www.richterzeitung.ch

Thomas Stadelmann / Andreas Lienhard / Anne Sanders

\title{
Gründung des Vereins «Schweizerisches Institut für Judikative»
}

Die Schweiz hat einen zusätzlichen Akteur im Bereich der Judikative: das «Schweizerische Institut für Judikative». Gegründet wurde der Trägerverein des Instituts im August dieses Jahres durch Akteure aus der Schweizer Judikative und der mit Justizforschung befassten Wissenschaft.

Beitragsart: News $\mathrm{CH}$

Zitiervorschlag: Thomas Stadelmann / Andreas Lienhard / Anne Sanders, Gründung des Vereins «Schweizerisches Institut für Judikative», in: «Justice - Justiz - Giustizia» 2020/4 
[1] Die Aufgabe des «Schweizerischen Instituts für Judikative» besteht darin, Funktion und Wirkungsweise der dritten Gewalt vertieft wissenschaftlich zu untersuchen sowie öffentlich und für die Forschung vermehrt zugänglich zu machen. Adressaten sind namentlich die Justiz, die Advokatur, die Öffentlichkeit, die Wissenschaft und die Politik.

[2] Das Institut ist Teil eines nationalen und internationalen Netzwerks. Es bietet Auskünfte, Expertisen und andere Dienstleistungen zu Fragen zur Judikative an. Darüber hinaus schafft es ein Forschungszentrum zur Judikative mit entsprechender Dokumentation. Mit dem Institut entsteht eine Einrichtung des Austauschs und der Wissensvermittlung im Rahmen wissenschaftlicher Tagungen in Kooperation und Absprache mit anderen Institutionen.

[3] Das Institut verfolgt einen interdisziplinären Ansatz. Neben dem Fokus auf der Rechtswissenschaft sollen insbesondere auch politik- und sozialwissenschaftliche Aspekte sowie Aspekte der Organisationslehre und der Ökonomie beleuchtet werden.

[4] Im Zentrum der Tätigkeiten werden Forschung und Dokumentation sowie Dienstleistungen stehen, als Ausfluss daraus soll - in Ergänzung zum bereits bestehenden Angebot - auch die Wissensvermittlung erfolgen.

[5] Folgende erste Aktivitäten sind bereits geplant oder in Vorbereitung:

- Auf der Website www.sifj.ch wird ein Verzeichnis geplanter oder im Entstehen begriffener juristischer Arbeiten zur Judikative - Habilitationen, Dissertationen, Masterarbeiten, CAS-Arbeiten - aufgebaut. Verfasser derartiger Arbeiten werden auf die Möglichkeit, ihre Arbeiten zu melden, hingewiesen (siehe https://sifj.ch/forschung/).

- Vor dem aktuellen Hintergrund der «Justizinitiative» (vgl. Geschäft des Bundesrates 20.061) sowie der Diskussion eines allfälligen Gegenvorschlages dazu (vgl. Parlamentarische Initiative RK NK 20.480), ist ein Policy-Paper in Arbeit, das eine Auslegeordnung von Optionen der Richterbestellung vornimmt. Informationen über neue Entwicklungen erfolgen auf der Website (sifj.ch) und via Twitter @SifjFur.

- Am Nachmittag des 12. März 2021 [save the date] werden wir an der juristischen Fakultät der Universität Basel eine Tagung zum Thema «Gewaltenteilung und Richterliche Unabhängigkeit in Europa und in der Schweiz - wo stehen wir ?» durchführen. Als Referierende und an den Podien nehmen teil: Prof. Dr. Stephan Breitenmoser, Universität Basel; Prof. em. Dr. Dr. h.c. mult. Dieter Grimm, ehem. Richter des Bundesverfassungsgerichts; Prof. Dr. Ulrich Meyer, Präsident des Schweizer Bundesgerichts; Prof. Dr. iur. Angelika Nußberger, ehem. Vizepräsidentin des EGMR; Prof. Dr. iur. Anne Sanders, Universität Bielefeld; Prof. Dr. iur. Peter Uebersax, Universität Basel; Prof. Dr. Dres. h.c. Andreas Voßkuhle, ehem. Präsident des Bundesverfassungsgerichts sowie Adrian Gasser, Initiativkomitee «Justizinitative», (für weitere Informationen siehe https://sifj.ch/wissens vermittlung/).

[6] Geleitet wird das Schweizerische Institut für Judikative von einem siebenköpfigen Vorstand, bestehend aus Dr. Arthur Brunner, Ersatzmitglied des Verwaltungsgerichts Zürich; PD Dr. Christoph Hurni, Bundesrichter (Lausanne); Prof. Dr. Regina Kiener, Universität Zürich; Prof. Dr. Andreas Lienhard, Universität Bern, Vizepräsident; Dr. Patrick M. Müller, Kantonsrichter Luzern; Prof. Dr. Anne Sanders, Universität Bielefeld, Vizepräsidentin, und Dr. h.c. Thomas Stadelmann, Bundesrichter (Luzern), Präsident. 
Dr. h.c. Thomas Stadelmann, Bundesrichter, Rechtsanwalt, Notar, dipl. Steuerexperte.

Prof. Dr. Andreas Lienhard, Ordinarius für Staats- und Verwaltungsrecht am Kompetenzzentrum für Public Management und am Institut für öffentliches Recht der Universität Bern.

Prof. Dr. Anne Sanders, Professorin für Bürgerliches Recht, Unternehmensrecht, das Recht der Familienunternehmen und Justizforschung an der Universität Bielefeld. 